\title{
Anaesthetic management of a unilateral adrenalectomy of an adrenocortical tumour in a dog
}

\author{
I.K. Wise ${ }^{1, *}$ and S. Boveri ${ }^{2}$ \\ ${ }^{1}$ University of Cambridge, Department of Clinical Veterinary Medicine, Madingley Road, Cambridge, UK \\ ${ }^{2}$ The University of Liverpool, School of Veterinary Science, Neston, UK
}

\begin{abstract}
Adrenalectomies in dogs are being more commonly performed, however anaesthetic management of such cases can be challenging due to the multiple aetiologies of adrenal tumours and the physiological role of adrenal glands. This case report describes the anaesthetic management of a dog with clinical signs of hyperadrenocorticism that underwent unilateral adrenalectomy via laparotomy and discusses anaesthetic preparedness, protocol selection and management of complications for dogs undergoing adrenalectomy.
\end{abstract}

Keywords: Adrenal neoplasia, Adrenalectomy, Canine.

\section{Introduction}

Adrenalectomy is the treatment of choice for canine adrenal tumours for a good long-term outcome (Lang et al., 2011). Surgical techniques for adrenalectomies in dogs are increasingly reported (Kyles et al., 2003; Jiménez Peláez et al., 2008; Lang et al., 2011; Massari et al., 2011; Gójska-zygner et al., 2012; Smith et al., 2012; Barrera et al., 2013; Naan et al., 2013). However, anaesthetic management of these cases has rarely been described (Kyles et al., 2003; Jiménez Peláez et al., 2008; Lang et al., 2011; Massari et al., 2011). Complications reported for adrenalectomies are hypotension, hypertension, tachycardia, ventricular arrhythmias and haemorrhage, with a reported incidence of 35 to $100 \%$ (Lang et al., 2011). Perioperative mortality rates for adrenalectomies have been reported as $6 \%$ for elective procedures increasing to $50 \%$ when performed in emergencies (Lang et al., 2011). These mortality rates are higher than that of $1.33 \%$ in dogs classified as American Society of Anesthesiologists (ASA) physical status 3-5 in the Confidential Enquiry into Perioperative Small Animal Fatalities (Brodbelt et al., 2008).

The most common primary adrenal tumours are adrenocortical adenomas or adenocarcinomas, and phaeochromocytomas (Massari et al., 2011). Less commonly, myelolipomas, aldosteronomas, deoxycorticosterone- or sex hormone- secreting adrenal tumours are found (Massari et al., 2011). Adrenal tumours may be endocrinologically active (Gójskazygner et al., 2012); thus the presenting clinical signs and the physiological response to anaesthesia and surgery may reflect the type of tumour present.

\section{Case Details}

A 6-year-old female neutered $33 \mathrm{~kg}$ Airedale was referred to the Queens Veterinary Hospital, University of Cambridge, for investigation of an 18-month history of lethargy, weight gain with abdominomegaly, polyuria, polydipsia (PU/PD) and polyphagia. There had been concurrent thinning of the coat with hyperpigmentation of the skin. On examination, the dog was quiet, alert and responsive with a leftsided head tilt and left-sided facial muscle weakness suggestive of facial nerve paralysis. Cardiorespiratory examination was unremarkable. Complete haematology and a comprehensive biochemistry profile showed the following significant abnormalities: PCV 60\% (reference range 37-55); total protein $84 \mathrm{~g} / \mathrm{dL}(60-80)$; urea $9.5 \mathrm{mmol} / \mathrm{L}$ (2.5-7.5); ALT $283 \mathrm{iu} / \mathrm{L}$ (14-67); ALP $561 \mathrm{iu} / \mathrm{L}$ (26-107); cholesterol $14.1 \mathrm{mmol} / \mathrm{L}$ (3.3-6.5). Urinalysis of a free catch sample showed isosthenuria (urine specific gravity 1.008). A right adrenal mass was the only abnormality detected by abdominal ultrasonography. A total body computer tomography scan with iodinated contrast confirmed the presence of a well circumscribed hyperplastic right adrenal gland and a hypoplastic left adrenal gland with no evidence of vascular infiltration or metastatic disease. The owners declined further clinical pathology tests. A right-sided adrenocortical tumour causing clinical signs of hyperadrenocorticism was presumptively diagnosed. The patient was scheduled for right adrenalectomy via laparotomy and was assigned an ASA physical status of 3 .

The patient was premedicated with medetomidine (1 $\mu \mathrm{g} \mathrm{kg}^{-1}$, Sedator; Dechra Veterinary Products, Skipton, UK) and methadone (0.3 $\mathrm{mg} \mathrm{kg}^{-1}$, Comfortan; Dechra Veterinary Products, Skipton, UK) intramuscularly (IM) followed by the placement of a $20 \mathrm{G}$ catheter in the cephalic vein. After preoxygenation, general anaesthesia was induced with propofol (PropoFlo Plus; Zoetis, Tadworth, UK) $2.7 \mathrm{mg} \mathrm{kg}^{-1}$ and diazepam 
(Diazemuls; Actavis UK Ltd, Barnstable, UK) $0.2 \mathrm{mg} \mathrm{kg}^{-1}$ intravenously (IV). After oro-tracheal intubation with a $10 \mathrm{~mm}$ diameter cuffed tube, anaesthesia was maintained with isoflurane (IsoFlo; Zoetis, Tadowrth, UK) in oxygen. Monitoring included electrocardiography (ECG), pulse oximetry $\left(\mathrm{SpO}_{2}\right)$, invasive blood pressure, oesophageal temperature, end-tidal (ET) gas analysis and capnography. Active warming was provided using a semi-conductive heating device (Hot Dog; Augustine Temperature Management, Eden Prairie, USA) to maintain normothermia.

In anticipation of intraoperative haemorrhage, an $18 \mathrm{G}$ catheter was placed in the contralateral cephalic vein to enable rapid fluid administration. Blood products, hetastarch and crystalloids were placed in theatre. Intravenous fluid therapy (IVFT) consisted of Hartmann's solution (Compound Sodium Lactate for Infusion BP; Baxter, Staines-upon-Thames, UK) administered at $5 \mathrm{ml} \mathrm{kg}^{-1} \mathrm{hr}^{-1}$ during surgical preparation, doubled during the surgical procedure. Intraoperative blood loss was monitored using a combination of haemoglobin concentration measurement in the suction system and the swab gravimetric method.

Atracurium (Tracrium; GlaxoSmithKline, Uxbridge, UK) $0.2 \mathrm{mg} \mathrm{kg}^{-1}$ was administered IV to facilitate the surgical approach. The neuromuscular blockade was monitored with accelerometry (TOF watch ${ }^{\mathrm{TM}}$; Wardray Premise, Thames Ditton, UK) placed across the ulnar nerve. Additional atracurium $0.1 \mathrm{mg} \mathrm{kg}^{-1} \mathrm{IV}$ was administered as needed during the procedure to maintain a train of four (TOF) reading of one. The dog was mechanically ventilated to maintain normocapnia. Analgesia was provided using lidocaine (Lidocaine 2\% BP; Hameln Pharmaceuticals Ltd, Gloucester, UK) and fentanyl (Sublimaze; Janssen-Cilag Ltd, High Wycombe, UK) IV infusions, respectively at $40 \mu \mathrm{g} \mathrm{kg}^{-1} \mathrm{~min}^{-1}$ and 2-15 $\mu \mathrm{g} \mathrm{kg}^{-1} \mathrm{hr}^{-1}$, both preceded by loading boluses of $1 \mathrm{mg} \mathrm{kg}^{-1}$ and $2 \mu \mathrm{g} \mathrm{kg}^{-1} \mathrm{IV}$ respectively. Paracetamol (Perfalgan; Bristol-Myers Squibb Pharmaceutical Ltd, Uxbridge, UK) $10 \mathrm{mg} \mathrm{kg}^{-1}$ was administered IV towards conclusion of surgery and bupivicaine (Marcain 0.5\% Polyamp Steripack; AstraZeneca, Luton, UK) $10 \mathrm{ml}$ was splashed on the rectus abdominus muscles once sutured. Cefuroxime (Zinacef; GlaxoSmithKline, Uxbridge, UK) $20 \mathrm{mg} \mathrm{kg}^{-1}$ IV was administered every 90 minutes throughout surgery.

Approximately 30 minutes after commencement of surgery, when the mass was being manipulated via blunt dissection around the adrenal capsule, the dog became acutely hypertensive (systolic $200 \mathrm{mmHg}$ and diastolic $110 \mathrm{mmHg}$ ) with an increased HR of $110 \mathrm{bpm}$ and premature supraventricular complexes. Thus a fentanyl bolus of $2 \mu \mathrm{g} \mathrm{kg}^{-1}$ IV was administered followed by an increased infusion rate of $15 \mu \mathrm{g} \mathrm{kg}^{-1} \mathrm{hr}^{-1}$. Methadone $0.3 \mathrm{mg} \mathrm{kg}^{-1} \mathrm{IV}$ was also administered. These interventions resolved the arrhythmia and reduced the systolic blood pressure to $100 \mathrm{mmHg}$. During further manipulation of the mass, a sudden increase in systolic pressure $(200 \mathrm{mmHg})$ occurred with concurrent severe bradycardia $(30 \mathrm{bpm})$ with third-degree atrioventricular blocks. Promotion of vasodilation by increasing the isoflurane percentage and administering acepromazine (ACP Injection; Novartis Animal Health, Basingstoke, UK) $5 \mu \mathrm{g} \mathrm{kg}^{-1}$ IV increased the heart rate to $60 \mathrm{bpm}$ and reduced the systolic blood pressure to between 80 and $90 \mathrm{mmHg}$. The patient remained stable for the remainder of anaesthesia as the right adrenal gland and associated tumour were excised en mass via further blunt dissection and vessel ligation.

Prior to recovery, a lumbosacral epidural injection of morphine (Morphine BP; Martindale Pharmaceuticals UK, Wockhart, UK) $0.15 \mathrm{mg} \mathrm{kg}^{-1}$ diluted with sterile saline to a volume of $0.12 \mathrm{ml} \mathrm{kg}^{-1}$ was administered and an 8 Fr Foley urinary catheter (Infusion Concept, Sowerby Bridge, UK) was placed. The neuromuscular blockade was not antagonized as two equal twitches in response to double burst stimulation were seen. Anaesthesia and surgery times were 290 minutes and 225 minutes, respectively.

The dog recovered from anaesthesia in the intensive care unit (ICU). Post-operative monitoring consisted of hourly assessments of pulse rate and quality, ECG, respiratory rate and pattern, Doppler blood pressure monitoring, mucous membrane colour and capillary refill time. Urinary output was monitored hourly and electrolytes levels were monitored every six hours for the first 24 hours. Pain was assessed every two hours using a modified Glasgow pain scale. If the pain score exceeded six, methadone $0.2 \mathrm{mg} \mathrm{kg}^{-1} \mathrm{IM}$ was prescribed as rescue analgesia. Paracetamol $10 \mathrm{mg} \mathrm{kg}^{-1}$ was continued per os twice daily and lidocaine $40 \mu \mathrm{g} \mathrm{kg}^{-1} \mathrm{~h}^{-1}$ IV was infused for 24 hours. Prednisolone (Prednicare; Animalcare, Nether Poppleton, UK) $0.2 \mathrm{mg} \mathrm{kg}^{-1}$ was administered once orally in the early postoperative period. Omeprazole, cefuroxime and vitamin A were prescribed by the attending clinicians. Two hours into the recovery period, the systolic blood pressure increased to $200 \mathrm{mmHg}$ without concurrent arrhythmias. Therefore, acepromazine (ACP tablets; Elanco Animal Health, Basingstoke, UK) $1 \mathrm{mg} \mathrm{kg}^{-1}$ was administered orally, which reduced the systolic pressure to $140 \mathrm{mmHg}$ within 30 minutes. The patient had no further complications and was discharged from intensive care the following day. Histopathological analysis defined the resected mass as an adrenocortical carcinoma.

\section{Discussion}

Although adrenalectomy is the preferred treatment for adrenal tumours in dogs (Lang et al., 2011), complications during anaesthesia and surgery are common and sometimes fatal. This case provides an 
opportunity to reflect on anaesthetic preparedness, protocol selection and management of possible complications that may occur in dogs undergoing anaesthesia for adrenalectomy.

A functional adrenocortical tumour may cause clinical signs of hyperadrenocorticism, such as $\mathrm{PU} / \mathrm{PD}$, abdominal enlargement, hypertension, prolonged healing, skin changes, lethargy, polyphagia and facial paralysis (Johnson and Norman, 2007). Uncommonly, adrenocortical tumours may also produce excess mineralocorticoids, causing clinical signs of Addison's disease. Phaeochromocytomas produce excess catecholamines which may cause hypertensive episodes. Phaeochromocytomas often invade adjacent blood vessels or organs, which may cause haemorrhage into the retroperitoneal space. Therefore, clinical signs of phaeochromocytoma are often nonspecific and include weakness, tachypnoea, collapse, tachyarrhythmias and seizures (Herrera et al., 2008). In our patient, the presence of many clinical signs of hyperadrenocorticism without evidence of hypertensive episodes or local tumour invasion lead to a preoperative presumptive diagnosis of an adrenocortical tumour producing excess cortisol. Further preoperative diagnostic testing such as adrenocorticotropic hormone stimulation testing and urine protein-to-creatinine ratio may have provided additional supporting evidence. As commonly occurs with adrenal tumours, a definitive diagnosis of adrenocortical adenocarcinoma was not made until postoperative histopathology was performed on the resected mass.

In patients suspected to have a phaeochromoytoma due to documented hypertensive episodes, preoperative stabilisation with phenoxybenzamine has been advocated to decrease perioperative mortality (Kyles et al., 2003; Herrera et al., 2008; Massari et al., 2011). Phenoxybenzamine, an $\alpha$-adrenergic antagonist, irreversibly binds to $\alpha 1$ and $\alpha 2$ adrenergic receptors to block the response to circulating catecholamines (Herrera et al., 2008). Preoperative treatment of human patients is for at least two weeks (Kyles et al., 2003), while the literature for dogs reports time frames between 7 and 120 days (Herrera et al., 2008). As our patient had no preoperative clinical signs of hypertension, phenoxybenzamine was not prescribed.

The most common intraoperative complication reported is haemorrhage, more likely when a cavotomy is required. Preoperative blood typing would have been required if our patient had received previous blood transfusions (Tocci and Ewing, 2009). Preoperative coagulation parameters may allow for preoperative management of any abnormalities detected. Hypercoagulability has classically been described as a consequence of hyperadrenocorticism. However, this has recently been disputed in dogs (Klose et al., 2011). This patient had no preoperative clinical signs of hyper- or hypo-coagulopathies, therefore coagulation parameters were not measured. Having pre-emptively placed large bore peripheral IV catheters in the patient and also the placement of resuscitative fluids in theatre meant that rapid treatment of major haemorrhage could occur. The swab gravimetric method has been reported to accurately quantify intraoperative haemorrhage in dogs (Lee et al., 2006). Therefore, the additional measurement of haemoglobin concentration of the suction fluid to approximate the volume of haemorrhage was likely unnecessary, as this technique is reportedly of similar accuracy to the gravimetric technique (Clark et al., 2010).

The ideal anaesthetic protocol for adrenalectomies should maintain homeostasis despite potential sudden physiological changes. Surgical manipulation of the adrenal gland can lead to a catecholamine surge and subsequent cardiovascular manifestations, regardless of the adrenal tumour type present (Lang et al., 2011). The anaesthetic protocol should also provide analgesia and muscle relaxation.

Premedication with a pure mu agonist such as methadone (Kyles et al., 2003; Massari et al., 2011) provides pre-emptive analgesia, sedation and a reduction in the required inhalant minimum alveolar concentration (MAC) with minimal cardiovascular effects. Previous studies report the use of an opioidbenzodiazepine combination in dogs undergoing adrenalectomy (Jiménez Peláez et al., 2008; Lang et al., 2011). Benzodiazepines cause minimal cardiovascular depression; however, in adult dogs the sedation can be unreliable and dysphoria may occur (Sanchez et al., 2013). Sedation with acepromazine may provide benefit to hypertensive patients due to the $\alpha-1$ adrenergic blockade it produces (Monteiro et al., 2007). However, the prolonged and irreversible duration of action of acepromazine may impede the treatment of hypotension during anaesthesia. In this case, medetomidine was selected to provide sedation and MAC reduction of isoflurane beyond that of an opioid alone (Lerche and Muir III, 2006). An additional benefit of medetomidine is the profound sympatholytic effect attributable to central $\alpha 2$-adrenoreceptor activation, which may partially counteract the effect of a catecholamine surge (Väisänen et al., 2002). The marked cardiovascular effects caused by $\alpha-2$ agonists such as decreased cardiac output and transient hypertension are dose-dependent and short acting, and an antagonist drug is available (Pypendop and Verstegen, 1998).

Previous studies describe the use of propofol (Jiménez Peláez et al., 2008), etomidate or propofol co-administered with midazolam (Lang et al., 2011) as induction agents prior to adrenalectomy. In a paper describing anaesthesia protocols for humans undergoing adrenalectomies, etomidate was avoided due to its ability to suppress the adrenocortical axis and 
thus production of mineralo- and gluco-corticosteroids (Domi and Sula, 2011). In contrast, Dabbagh et al. (2009) reported infusing etomidate to control the clinical signs of hyperadrenocorticism preoperatively. Our choice of co-induction with propofol and diazepam has been described in the veterinary literature (Robinson and Borer-Weir, 2013; Sanchez et al., 2013), although the only benzodiazepine achieving a significant reduction in propofol dose has been midazolam. However, most patients in the study from Robinson and Borer-Weir (2013) were healthy dogs of ASA status 1 or 2, with an insufficient number of ASA 3 dogs in the treatment group to speculate association with ASA category. In our clinical experience, benzodiazepenes as co-induction agents at lower doses than those published often reduce the induction agent dose required in debilitated patients. All published reports utilise inhalant techniques for the maintenance of anaesthesia (Jiménez Peláez et al., 2008; Lang et al., 2011). Isoflurane was used in this case as the maintenance agent because sevoflurane was unavailable. The acute episode of severe vasoconstriction, hypertension and reflex bradycardia that occurred during adrenal gland manipulation was likely due to a catecholamine surge. The vasodilatory effect of isoflurane was utilised to manage this event. Ideally, anti-hypertensive drugs should be short-acting and titratable to effect. The use of sevoflurane may have allowed for more rapid changes in ET agent concentrations due to its lower blood-gas coefficient (Lopez et al., 2009). Other reportedly used vasoactive drugs such as phentolamine (Kyles et al., 2003), a short acting competitive $\alpha-1$ adrenergic blocking drug, or sodium nitroprusside (SNP) (Kyles et al., 2003; Lang et al., 2011) were unavailable. One study reported that the vasodilation induced by isoflurane was advocated to maintain better overall tissue perfusion than SNP (Humm et al., 2007). On this occasion, the short action of phentolamine would have been more suitable than acepromazine to provide additional vasodilation. Acepromazine likely contributed to the subsequent mild hypotension. Furthermore, the long and irreversibly action of acepromazine may have impeded management of blood pressure were it to worsen (Monteiro et al., 2007). If the reflex bradycardia had not responded to the induction of vasodilation, treatment with an anticholinergic such as atropine would have been indicated.

Tachyarrhythmias commonly occur due to adrenal gland manipulation, nociceptive input or in response to hypotension. Supraventricular tachycardias (SVT) and ventricular arrhythmias (Kyles et al., 2003; Lang et al., 2011) have been reported during adrenalectomy. In this instance, opioids successfully treated the supraventricular tachycardia by reducing both sympathetic tone and nociception as reported in older studies. A more recent paper (Garofalo et al., 2008) failed to demonstrate a protective effect of remifentanil against ventricular arrhythmias induced by adrenaline in halothane-anaesthetised dogs. Myocardial sensitization to catecholamine-induced arrhythmias by halothane as used in Garofalo's study may have made tachyarrythmias more difficult to treat compared to isoflurane. The second-line treatment for SVT would have been ultra short-acting beta-blockers such as esmolol (Kyles et al., 2003; Lang et al., 2011). If ventricular arrhythmias had occurred, treatment with higher doses of lidocaine or with procainamide would have been indicated (Lang et al., 2011).

Multimodal analgesia was provided with the co-administration of fentanyl and lidocaine; the latter also for its MAC sparing (Valverde et al., 2004) and antiarrhythmic properties (Bruchim et al., 2012). Opioids have been used in all published studies, with hydromorphone, methadone, morphine and fentanyl infusions reported (Jiménez Peláez et al., 2008; Lang et al., 2011). Nonsteroidal anti-inflammatory drugs (NSAIDs) were avoided as they may precipitate gastrointestinal ulceration in patients with excess glucocorticoids (Kukanich et al., 2012). To avoid this, paracetamol was preferred as an adjunctive analgesic in this case (Benitez et al., 2015).

Neuromuscular blockade may facilitate surgical approaches to deep structures during laparotomies (Clarke et al., 2014) but has only been described in one veterinary paper describing adrenalectomies (Lang et al., 2011). In a case report of two human adrenalectomies (Domi and Sula, 2011), cis-atracurium was selected for its short duration of action and plasma degradation; for similar reasons, atracurium was administered to our patient.

Post-operative complications occur with high incidence following adrenalectomies (30\% according to Lang et al. (2011)), necessitating close monitoring in ICU settings. Reported complications include hypo- or hyper-tension, arrhythmias, vomiting, oesophagitis, seizures, pancreatitis, acute renal failure, haemoperitoneum, hyperthermia, hypokalaemia, tachypnoea and cardiopulmonary arrest (Kyles et al., 2003; Lang et al., 2011 Gójska-zygner et al., 2012). Many complications relate to inadequate function of the remaining adrenal gland, leading to acute adrenal insufficiency and crisis. Corticoid administration using various protocols in the perioperative period features in all published studies. Most commonly reported is glucocorticoid supplementation with dexamethasone once the adrenal tumour is removed, followed by administration of prednisolone in the recovery period (Kyles et al., 2003; Lang et al., 2011). In this case, as commonly occurs, it was unknown whether the remaining hypoplastic adrenal gland was capable of producing sufficient corticoids postoperatively. Therefore, a single postoperative dose of prednisolone 
was administered to provide additional glucocorticoid support for recovery from the physiological stress of anaesthesia and surgery. Close monitoring of vital signs was undertaken to detect signs of an adrenal crisis, such as cardiovascular shock or vomiting. Serum electrolytes were measured every six hours in the early postoperative period to identify any acute mineralocorticoid deficiency and prompt treatment if required. The only identified postoperative complication in our case was mild hypertension, which responded to the administration of acepromazine.

In conclusion, the anaesthetic management for adrenalectomies is challenging because of the high incidence of severe complications. Agood understanding of the physiological derangements that may be induced by both the tumour itself and the surgical procedure is required. Adequate preparations for anticipated problems, including availability of resuscitative fluids and short-acting drugs, will minimize patient morbidity and mortality.

\section{References}

Barrera, J.S., Bernard, F., Ehrhart, E.J., Withrow, S.J. and Monnet, E. 2013. Evaluation of risk factors for outcome associated with adrenal gland tumors with or without invasion of the caudal vena cava and treated via adrenalectomy in dogs: 86 cases (19932009). J. Am. Vet. Med. Assoc. 242(12), 1715-1721.

Benitez, M.E., Roush, J.K., McMurphy, R., KuKanich, B. and Legallet, C. 2015. Clinical efficacy of hydrocodone-acetaminophen and tramadol for control of postoperative pain in dogs following tibial plateau leveling osteotomy. Am. J. Vet. Res. 76(9), 755-762.

Brodbelt, D.C., Blissitt, K.J., Hammond, R.A., Neath, P.J., Young, L.E., Pfeiffer, D.U. and Wood, J.L. 2008. The risk of death: Confidential Enquiry into Perioperative Small Animal Fatalities. Vet. Anaesth. Analg. 35(5), 365-373.

Bruchim, Y., Itay, S., Shira, B.H., Kelmer, E., Sigal, Y., Itamar, A. and Gilad, S. 2012. Evaluation of lidocaine treatment on frequency of cardiac arrhythmias, acute kidney injury, and hospitalization time in dogs with gastric dilatation volvulus. J. Vet. Emerg. Crit. Care 22(4), 419-27.

Clark, L., Corletto, F. and Garosi, L.S. 2010. Comparison of a method using the Hemocue near patient testing device with a standard method of haemorrhage estimation in dogs. Vet. Anaesth. Analg. 37(1), 44-47.

Clarke, K.W., Trim, C.M. and Hall, L.W. (eds). 2014. Veterinary Anaesthesia, $11^{\text {th }}$ ed. London: Saunders Elsevier, pp: 169-194.

Dabbagh, A., Sa'adat, N. and Heidari, Z. 2009. Etomidate infusion in the critical care setting for suppressing the acute phase of Cushing's syndrome.
Anesth. Analg. 108(1), 238-239.

Domi, R. and Sula, H. 2011. Cushing syndrome and the anesthesiologist, two case reports. Indian J. Endocrinol. Metab. 15(3), 209-213.

Garofalo, N.A., Teixeira-Neto, F.J., Schwartz, D.S., do Carmo, F., Vailati, M. and Steagall, P.V.M. 2008. Effects of the opioid remifentanil on the arrhythmogenicity of epinephrine in halothaneanesthetized dogs. Can. J. Vet. Res. 72(4), 362-366.

Gójska-zygner, O., Lechowski, R. and Zygner, W. 2012. Functioning unilateral adrenocortical carcinoma in a dog. Can. Vet. J. 53(6), 623-625.

Herrera, M.A., Mehl, M.L., Kass, P.H., Pascoe, P.J., Feldman, E.C. and Nelson, R.W. 2008. Predictive factors and the effect of phenoxybenzamine on outcome in dogs undergoing adrenalectomy for pheochromocytoma. J. Vet. Intern. Med. 22(6), 1333-1339.

Humm, K.R., Senior, J.M., Dugdale, A.H. and Summerfield, N.J. 2007. Use of sodium nitroprusside in the anaesthetic protocol of a patent ductus arteriosus ligation in a dog. Vet. J. 173(1), 194-196.

Jiménez Peláez, M., Bouvy, B.M. and Dupré, G.P. 2008. Laparoscopic adrenalectomy for treatment of unilateral adrenocortical carcinomas: technique, complications, and results in seven dogs. Vet. Surg. 37(5), 444-453.

Johnson, C. and Norman, E.J. 2007. 'Endocrine disease', in Seymour, C. and Duke-Novakovski, T. (eds), BSAVA Manual of Canine and Feline Anaesthesia and Analgesia, 2 ${ }^{\text {nd }}$ edn. Gloucester, UK: British Small Animal Veterinary Association.

Klose, T.C., Creevy, K.E. and Brainard, B.M. 2011. Evaluation of coagulation status in dogs with naturally occurring canine hyperadrenocorticism. J. Vet. Emerg. Crit. Care 21(6), 625-632.

KuKanich, B., Bidgood, T. and Knesl, O. 2012. Clinical pharmacology of nonsteroidal anti-inflammatory drugs in dogs. Vet. Anaesth. Analg. 39(1), 69-90.

Kyles, A.E., Feldman, E.C., De Cock, H.E., Kass, P.H., Mathews, K.G., Hardie, E.M., Nelson, R.W., Ilkiw, J.E. and Gregory, C.R. 2003. Surgical management of adrenal gland tumors with and without associated tumor thrombi in dogs: 40 cases (1994-2001). J. Am. Vet. Med. Assoc. 223(5), 654-662.

Lang, J.M., Schertel, E., Kennedy, S., Wilson, D., Barnhart, M. and Danielson, B. 2011. Elective and emergency surgical management of adrenal gland tumors: 60 cases (1999-2006). J. Am. Anim. Hosp. Assoc. 47(6), 428-435.

Lee, M.H., Ingversten, B.T., Kirpensteijn, J., Jensen, A.L. and Kristensen, A.T. 2006. Quantification of surgical blood loss. Vet. Surg. 35(4), 388-393. 
Lerche, P. and Muir III, W.W. 2006. Effect of medetomidine on respiration and minimum alveolar concentration in halothane- and isofluraneanesthetized dogs. Am. J. Vet. Res. 67(5), 782-789.

Lopez, L.A., Hofmeister, E.H., Pavez, J.C. and Brainard, B.M. 2009. Comparison of recovery from anesthesia with isoflurane, sevoflurane, or desflurane in healthy dogs. Am. J. Vet. Res. 70(11), 1339-1344.

Massari, F., Nicoli, S., Romanelli, G., Buracco, P. and Zini, E. 2011. Adrenalectomy in dogs with adrenal gland tumors: 52 cases (2002-2008). J. Am. Vet. Med. Assoc. 239(2), 216-221.

Monteiro, E.R., Teixeira Neto, F.J., Castro, V.B. and Campagnol, D. 2007. Effects of acepromazine on the cardiovascular actions of dopamine in anesthetized dogs. Vet. Anaesth. Analg. 34(5), 312-321.

Naan, E.C., Kirpensteijn, J., Dupré, G.P., Galac, S. and Radlinsky, M.G. 2013. Innovative approach to laparoscopic adrenalectomy for treatment of unilateral adrenal gland tumors in dogs. Vet. Surg. 42(6), 710-715.

Pypendop, B.H. and Verstegen, J.P. 1998. Hemodynamic effects of medetomidine in the dog: a dose titration study. Vet. Surg. 27(6), 612-622.

Robinson, R. and Borer-Weir, K. 2013. A dose titration study into the effects of diazepam or midazolam on the propofol dose requirements for induction of general anaesthesia in client owned dogs, premedicated with methadone and acepromazine. Vet. Anaesth. Analg. 40(5), 455-463.

Sanchez, A., Belda, E., Escobar, M., Agut, A., Soler, M. and Laredo, F.G. 2013. Effects of altering the sequence of midazolam and propofol during co-induction of anaesthesia. Vet. Anaesth. Analg. 40(4), 359-366.

Smith, R.R., Mayhew, P.D. and Berent, A.C. 2012. Laparoscopic adrenalectomy for management of a functional adrenal tumor in a cat. J. Am. Vet. Med. Assoc. 241(3), 368-372.

Tocci, L.J. and Ewing, P.J. 2009. Increasing patient safety in veterinary transfusion medicine: an overview of pretransfusion testing. J. Vet. Emerg. Crit. Care 19(1), 66-73.

Väisänen, M., Raekallio, M., Kuusela, E., Huttunen, P., Leppäluoto, J., Kirves, P. and Vainio, O. 2002. Evaluation of the perioperative stress response in dogs administered medetomidine or acepromazine as part of the preanesthetic medication. Am. J. Vet. Res. 63(7), 969-975.

Valverde, A., Doherty, T.J., Hernández, J. and Davies, W. 2004. Effect of lidocaine on the minimum alveolar concentration of isoflurane in dogs. Vet. Anaesth. Analg. 31(4), 264-271. 